\title{
Sensors Based on Optical Fibre Microwires Coils and Related Resonators
}

\author{
Gilberto Brambilla \\ Optoelectronics Research Centre, University of Southampton, Southampton, SO17 1BJ \\ gb2@orc.soton.ac.uk
}

\begin{abstract}
University of Southampton on the use of microfiber coils for sensing.

OCIS codes: (060.2370) Fiber optics sensors; (060.2340) Fiber optics components
\end{abstract}

Optical fibre microwires (OFMs) are tapered optical fibres with size comprised between $100 \mathrm{~nm}$ and $10 \mu \mathrm{m}$. In the recent years OFMs and their related devices and sensors attracted increasing attention [1,2], because of their remarkable optical and mechanical properties, including: high strength, flexibility, large evanescent fields, tight confinement, configurability, and biocompatibility. When OFMs are bent to form loops or coils, the mode propagating in them experiences coupling between different OFM sections and resonating structures can be easily formed [1,2]. Resonators with Q-factors in excess of $2 \cdot 10^{5}$ have been recently demonstrated. Several types of resonators manufactured from OFM have been demonstrated to date, including loop, knot, reef knot, racetrack and coil resonators. Because of the way they are manufactured, OFM resonators do not have a geometry stable over long periods of time (for example coil relies on surface charges to maintain the different parts of OFM in contact) and above all they suffer from mechanical and optical degradation. Embedding has been proved to be a valuable solution for both temporal stability and degradation. Yet, OFMs have been proposed for a wide range of sensors, both in their linear or resonant configuration [3]. At the University of Southampton, non-resonant microcoils have been proposed as absorption chemical sensor and a current sensor [4], while OFM resonators were used as refractometric sensors.

The absorption chemical sensor was fabricated tapering a standard telecom optical fiber to $\mathrm{r}=1 \mu \mathrm{m}$ over $\mathrm{L}=9 \mathrm{~mm}$ and then coiling it onto an expendable $\mathrm{d}=1 \mathrm{~mm}$ poly-methyl-methacrylate (PMMA) support rod which was embedded in Teflon and then dissolved in acetone. Before embedding, the PMMA rod extremities were connected to two Teflon tubes $\left(\mathrm{d}_{\mathrm{Int}}=1 \mathrm{~mm}, \mathrm{~d}_{\mathrm{Ext}}=1.2 \mathrm{~mm}\right)$, which provided the microfluidic channel to deliver and collect the analyte from the sensor. The Langmuir equation, which describes the adsorption of molecules on surfaces, precisely described the dependence of absorption on analyte concentration.

The current sensor was based on the Faraday effect, which relates the intensity of magnetic field induced by the applied current to the change in polarization azimuth of the propagating optical field. The passage of current through the copper wire is associated with an increase in the magnetic field, thus with a change in the polarization state of the mode guided in the OMF. The polarization change is detected as a change in intensity at the detector. A linear relationship was recorded between polarization rotation and current in the range 0-19Amps for AC frequencies between 1 and $2 \mathrm{kHz}$. The sensor responsivity was $16.8 \pm 0.1 \mu \mathrm{rad} / \mathrm{A}$, similar to the values recorded at sub-hertz frequencies.

The refractometric sensor exploited the microcoil resonating properties [5]. By opportunely choosing the microfiber diameter and coil distance, the resonator spectral response [6] and the fraction of the mode extending into the microfluidic channel were optimised. The relation between the resonance shift and the fluid refractive index gave a sensor responsivity of $40 \mathrm{~nm} / \mathrm{RIU}$. Simulations showed that the recorded responsivity corresponded to negligible polymer thicknesses, meaning that the thickness of the polymer layer separating the microcoil from the OFM microfluidic channel is really minuscule.

\section{References}

[1] L. Tong, R.R. Gattass, J.B. Ashcom, S. He, J. Lou, M. Shen, I. Maxwell1 and E. Mazur, "Subwavelength-diameter silica wires for low-loss optical wave guiding," Nature 426, 816-819 (2003).

[2] G. Brambilla, “Optical fibre nanowires and microwires: a review,” J. Opt. 12043001 (2010).

[3] G. Brambilla, "Optical fibre nanotaper sensors", Opt. Fib. Technol. 16, 331-342 (2010).

[4] M. Belal, Z. Song, Y. Jung, G. Brambilla, and T. P. Newson, "Optical fiber microwire current sensor," Opt. Lett. 35, 3045-3047 (2010).

[5] F. Xu and G. Brambilla, "Demonstration of a refractometric sensor based on optical microfiber coil resonator," Appl. Phys. Lett. 92, 101126

(2008).

[6] F. Xu, P. Horak, G. Brambilla, "Optimized Design of Microcoil Resonators," J. Lightwave Technol. 25, 1561-1567 (2007). 